\title{
Latidens salimalii (Endemic, Endanger Fruit Bat) A Reliable Propagator of Endemic Trees of Southern Western Ghats
}

\author{
Juliet Vanitharani \\ Bat Research Laboratory, Zoology Department and Research Centre, Sarah Tucker College (Autonomous) Tirunelveli, Tamilnadu \\ 627007, India
}

\begin{abstract}
Salim Ali's fruit bat (Latidens salimalii) is Endemic to Southern Western Ghats, India and is classified as Endangered by IUCN. Latidens is morphologically adapted to play a major role in the propagation of economically important trees; some of them are endemic to southern Western Ghats. This paper describes and documents, the bat's ecology, foraging behavior and food selection. The study is based on the dietary details of the discovered populations of L. salimalii in the Agasthiyar hill range and the High Wavy Mountains of southern Western Ghats, India. Seeds, partially eaten fruits and seedlings germinated on the floor of the feeding roosts confirm this bat forage for fruits among relatively tall trees of evergreen forests in an elevation above 900 meter. Mist netting below and above canopy near the fruiting and flowering trees of their foraging area confirmed their interaction with certain endemic tree species. Their species specific dietary preferences immensely help to restore and bring back the natural forest community structures.
\end{abstract}

Key words: Endemic, foraging, feeding roosts, propagation, restoration.

\section{Introduction about Latidens Salimalii}

In 1948, a fruit bat, believed at the time to be Cynopyterus sphinx, was collected by Angus Hutton from the High Wavy Mountains in the Madurai District of Tamil Nadu, southern India. The specimen was placed in the collection of the Bombay Natural History Society and it was not until its re-examination in 1970 by Kitty Thonglongya that a number of its characteristics were found to be incompatible with those of $C$. sphinx. As a result, the specimen was assigned to a new genus and species, Latidens salimalii Thonglongya, 1972, so named on account of its broad cheek teeth and in honor of the Indian ornithologist Dr. Salim Ali. The High Wavy Mountains remained the only recorded distributional record of $L$. salimalii until 1999, when its presence was recorded, in the Kalakkad-Mundanthurai Tiger Reserve, Tamil Nadu (Vanitharani 2003, 2005, 2006, 2007a; Vanitharani

Corresponding author: Juliet Vanitharani, professor, research field: bat research and biodiversity conservation of western ghats. E-mail: jvanitharani@gmail.com. and Jeya Praba 2011;Vanitharani et.al. 2004, 2011), thereby extending its range between 110 and $160 \mathrm{~km}$ southwards (Map1). L. salimalii is endemic to southern Western Ghats, India (Bates and Harrison 1997) and is classified as Critically Endangered by the 2003 IUCN Red List of Threatened Species. Like other Pteropods $L$. salimalii also make local migrations in search of ripe fruit and also migrate seasonally to areas of better food resources (Marshall 1983).

L. salimalii depends heavily on plant resources throughout the year (Plate 1). The feeding habits and mobility of the bat play important roles as pollinators and seed dispersers in the evergreen forest ecosystem of southern Western Ghats. Fruit bats' mutualistic interaction with plant community helps in forest restoration (Omaston, 1965; Janzen, 1970; Thomas, 1982; Howe and Smallwood 1982; Augsburger 1984, Vanitharani 2007b; Vanitharani et. al. 2007 ). Bat-plant interactions include benefits such as balanced nutrition for bats, quick fertilization of ovules, and promotion of gene exchange, to escape specialized 


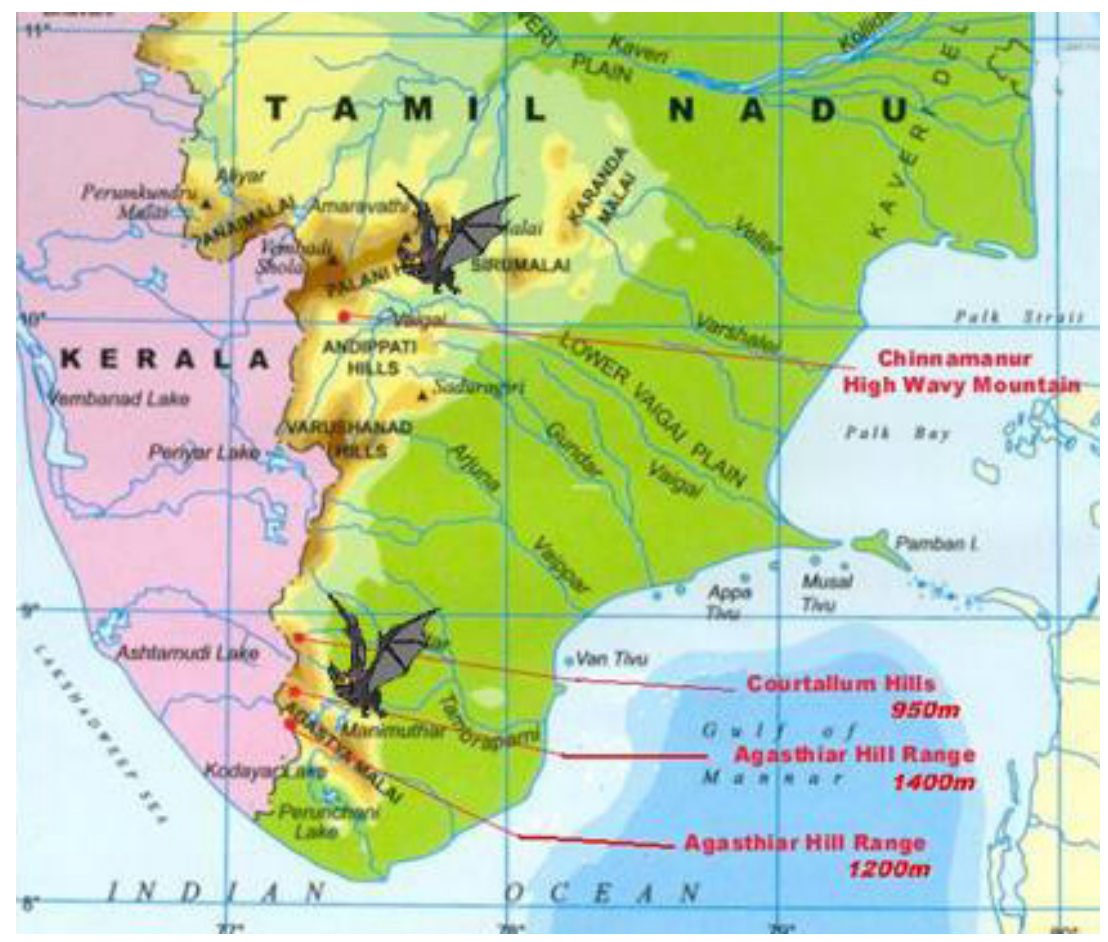

Map1 Study locations of $L$. salimalii confined to the Tamil Nadu part of southern Western Ghats.

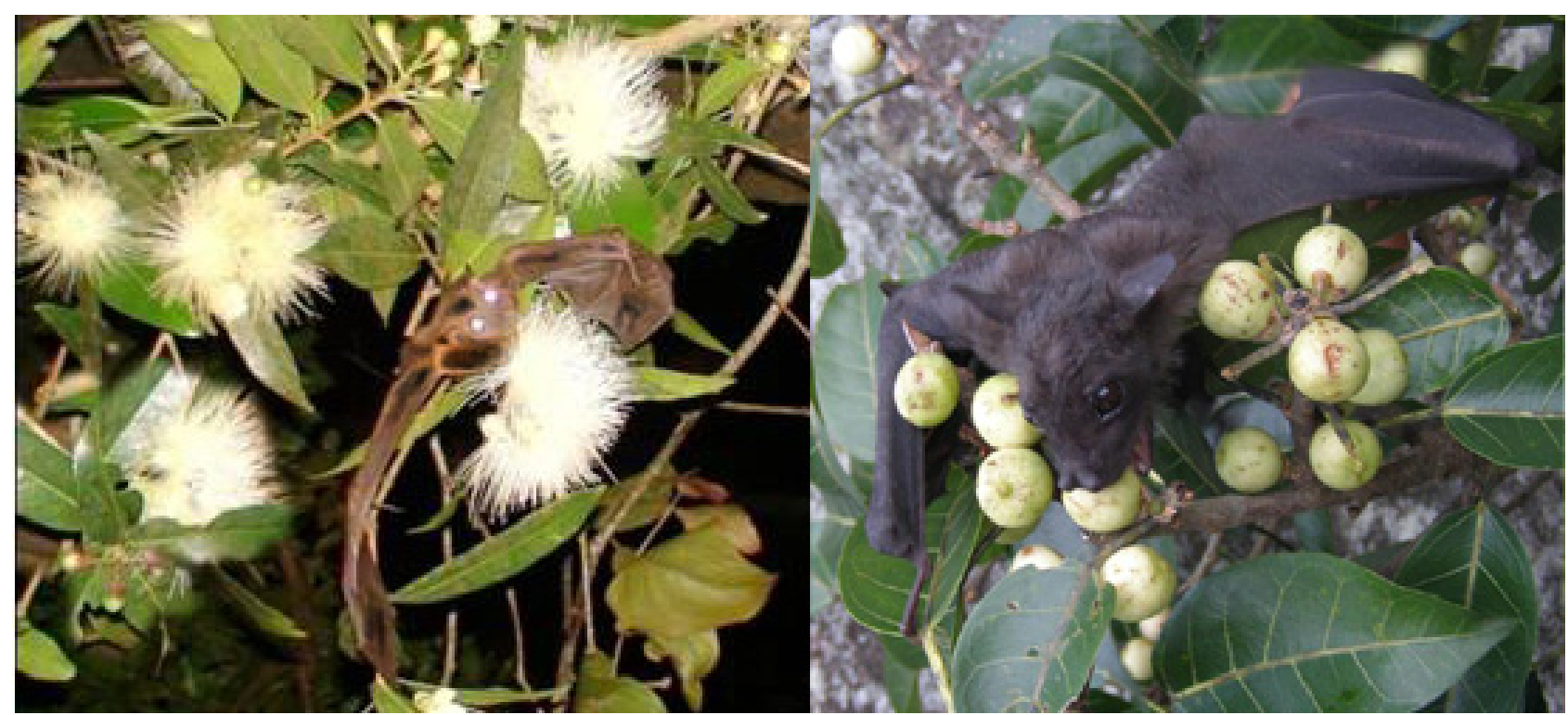

Plate 1 L. salimalii the reliable seed disperser and pollinator: inter action with Ficus asperima and Syzygium mundagam.

seed predators and enhance colonization of plant community. This interactions intern the forest structure with increased and improved biodiversity (Vanitharani et.al. 2011).

\section{Materials and Methods}

Field studies were carried out at severely fragmented, L. salimalii distributed areas of southern Western Ghats, endemic to Tamil Nadu \{Ambalam ( $8^{\circ} 36.42^{\prime} \mathrm{N}$ lat. $77^{\circ} 18.38^{\prime} \mathrm{E}$ long; elevation 1,460ft), Sengaltheri $\left(8^{\circ} 32.030^{\prime} \mathrm{N}\right.$ lat. $77^{\circ} 26.877^{\prime} \mathrm{E}$ long; elevation 2,814ft of Agasthiyamalai and Megamalai (09³9.835' N lat. $077^{\circ} 21.763^{\prime} \mathrm{E}$ long, elevation: $4,995 \mathrm{ft}$ ) day roosting caves. The feed analysis studies 


\section{Endemic Trees of Southern Western Ghats}

were made in feeding roosts located at Therkumalai ( $8^{\circ} 53.838^{\prime} \mathrm{N}$ lat. $77^{\circ} 15.248^{\prime} \mathrm{E}$ long; elevation: 2,412ft) deserted British Raj's building and Kuthiraivettai cave ( $8^{\circ} 41.283^{\prime} \mathrm{N}$ lat. $77^{\circ} 31.098^{\prime} \mathrm{E}$ long; elevation: 3,343ft) Theni Pudhukadu, Venniyar $\left(09^{\circ} 38.380^{\prime} \mathrm{N}\right.$ lat. $077^{\circ} 22.011^{\prime}$ E long; elevation: 5,009ft) and Kardana cave $\left(09^{\circ} 41.610^{\prime} \mathrm{N}\right.$ lat. $077^{\circ} 24.232^{\prime} \mathrm{E}$ long, elevation: $3,709 \mathrm{ft})\}$.

The morphometry study and digital recording were done on adult mist netted bats caught near the day roost cave entrances (Avinet NY 2.6 x 9m Nylon net with $88 \mathrm{~mm}$ mesh size) and the bats were released back immediately to lessen the stress and disturbance. Morphological measurements of the bats were taken by following the standard techniques of Bates and Harrison (1997) and aerodynamic predictions were documented based on the calculations of Norberg and Rayner (1987).

Once in fortnight plastic sheets were spread on the floor of the feeding roost sites to collect the remains of the food items and faeces. Examination of these left outs, spit outs, faeces and the germinated seedlings helped to find out the feeding habit and the dietary selection (Plate 2). Latidens interacting plants were identified by careful examination of the plant's characters by the botanist, checking with the literatures and the authentic herbarium of southern Western Ghats flora, available at the Research center of the Central
Council for Research in Ayurvetha and Siddha (Govt. of India), Palayamkottai. Further confirmation about $L$. salimalii's interaction was done by mist netting around the identified fruiting and flowering trees distributed in the foraging area of the bat.

\section{Results}

L. salimalii live a colonial life with a group consisting of a few hundreds and they roost diurnally in wide mouthed caves with dark, narrow branched chambers, located adjacent to riverbeds above 900 metres within the Hill complex (Plate 3). Being very sensitive to disturbance Latidens selects day roosts far away from the feeding sites. Home range consists minimum of $10 \mathrm{kms}$ radius around the day and night roosting areas. They show migration and shifting of roosting sites depending on the food source availability.

The plants benefited for dispersal and pollination through L. salimalii, together with indications of their economic importance are given in Table 1. Selection of food by L. salimalii primarily depends on their skull and wing morphology. The robust wide skull with wide open jaws, higher coronoid process for the attachment of larger jaw muscle and the wide palate with larger breadth enable the bat to pluck and transport larger fruits. The dentition is thicker with lager canine (Plate 4 Table 2a, b). Latidens has narrow

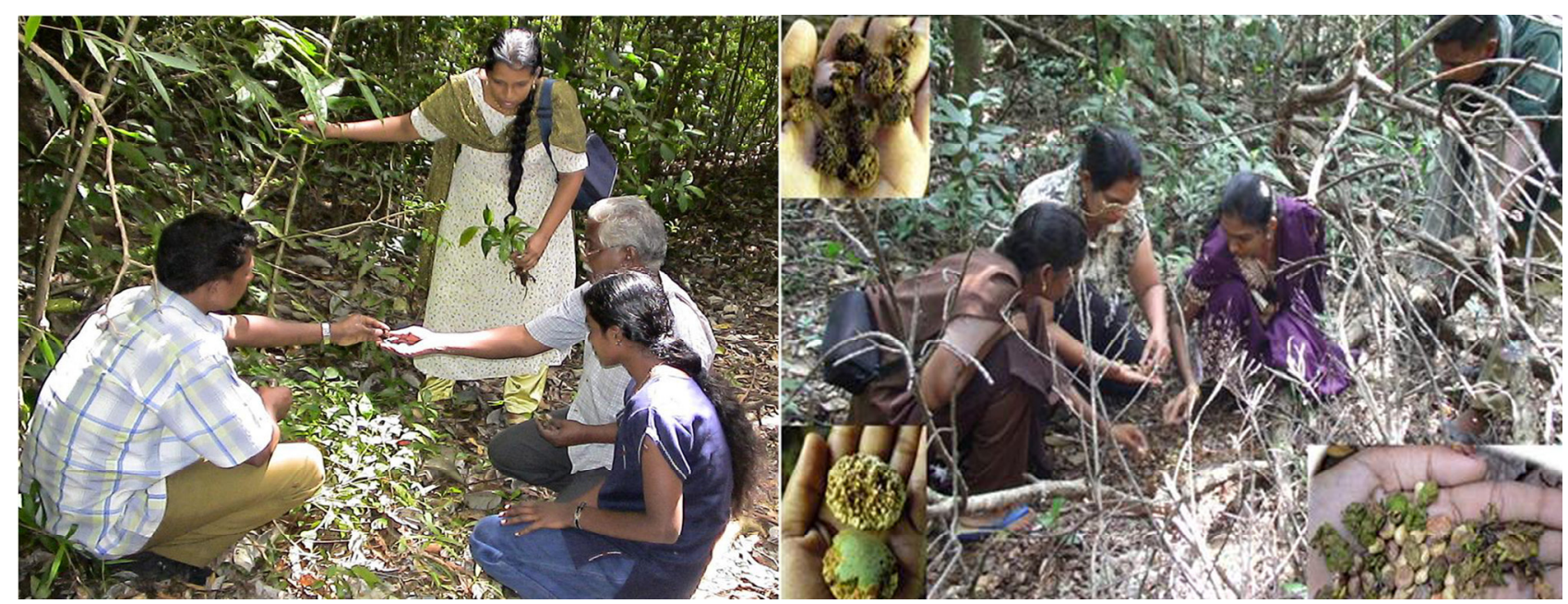

Plate 2 Portrays the location of a few feeding roosts in the forest area with seeds and seedlings of rare and endemic plant species. 


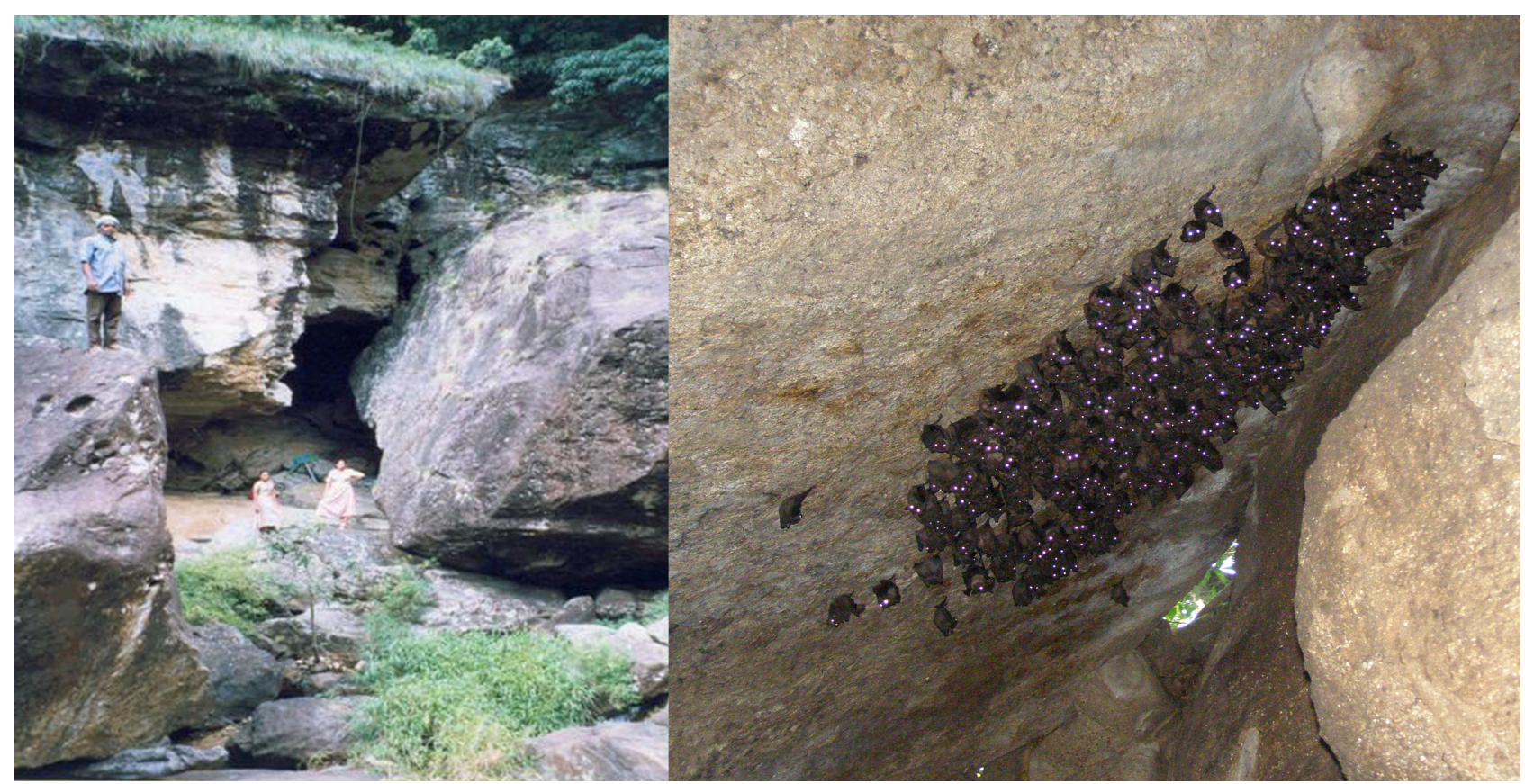

Plate 3 L. salimalii colony from a caves located among the rocks adjacent to pachayar river of Sengaltheri above 900 metres within the Hill complex of southern Western Ghats.

and shorter wings, with small wing area, High wing loading similar to Molossid bat species, an indication for the possession of high flight speed. Low aspect ratio indicates they are very agile and the rounded wing tip provides high manoeuvrability. Absence of uropatagium gives freedom of hind limb to crawl over vegetation while selection of fruits and nectar from the wide open bat preferred flowers (Plate 4 Table 3a, b).

L. salimalii does not feed while on wings. Consume fruits in secluded night roosts. Table $4 \mathrm{a}$, b portrays the location of a few feeding roosts in the forest area with seeds and seedlings of rare and endemic plant species propagated by $L$. salimalii. Computerized unit of GC-MS studies of saliva gut and faecal sample has proved the chemical treatment is given by the bats to the during food processing.

\section{Discussion}

L. salimalii is found to play a significant role in the evolution and maintenance of the tropical, forest ecosystem of the southern Western Ghats. The ecomorphology, feeding and foraging behaviour and the bat-plant species specific interactions confirm $L$. salimalii is a reliable seed disperser and pollinator.

\subsection{Foraging Pattern and Food Selection}

L. salimalii's foraging behaviour enable the bat to exploit a variety of foraging niches inaccessible to other mammals. The foraging ground lies among the semi ever green, hill top evergreen and the riparian forests of higher elevations (above $900 \mathrm{~m}$ above sea level). It flies long distances nightly to locate food, (Vanitharani's unpublished radiotelemetry data). During their wide nightly foraging routes they assess and search a number of plants for the distribution of ripe fruits (Fleming, 1982 and 1988).

The quality of seed dispersal depends on seed and fruit characteristics and seed-disperser's assistance in the dissemination of diaspor to far away locations with suitable micro-sites required for seed germination (Kumaravelu, 2007). Since the nutritional requirement of fruit bat species differs in accordance to their physiology and flight patterns each of them has specific core plant species to satisfy their dietary requirements and they focus their feeding and foraging efforts on specific suites of fruits. Thus they mediate resource partitioning in the forest ecosystem (Utzurrum 1995; Willig et al., 1993). This behaviour 
Table 1 Plants propagated through the ecosystem services of L. Salimalii, (the core species) together with indications of their economic importance.

\begin{tabular}{|c|c|c|c|c|c|c|c|}
\hline $\begin{array}{l}\text { Name of Core Plant } \\
\text { Species }\end{array}$ & Flower & Fruit & Seedlings & Location & Tree height (m) & Medicinal importance & Ecological importance \\
\hline $\begin{array}{l}\text { Alseodaphne } \\
\text { somecarpifolia } \\
\text { (Lauraceae) }\end{array}$ & - & - & $\sqrt{ }$ & & Large tree $28 \mathrm{~m}$ & Fruits: Treat leach bite. & $\begin{array}{l}\text { Fruit: Feed for } \\
\text { frugivorous bats and birds }\end{array}$ \\
\hline \begin{tabular}{|l|} 
Atalantia \\
monophylla \\
(Rutaceae) \\
\end{tabular} & - & $\sqrt{ }$ & - & & Small tree $15 \mathrm{~m}$ & $\begin{array}{l}\text { Fruits: oil to treat chronic } \\
\text { rheumatism }\end{array}$ & $\begin{array}{l}\text { Hard wood: substitute for } \\
\text { boxwood. Fruits are food } \\
\text { for }\end{array}$ \\
\hline $\begin{array}{l}\text { Bhesa indica (Bedd.) } \\
\text { Ding (Celastraceae) }\end{array}$ & - & $\sqrt{ }$ & - & & Large tree $30 \mathrm{~m}$ & & $\begin{array}{l}\text { Timber: suitable for } \\
\text { medium construction, } \\
\text { furniture, veneer and } \\
\text { plywood. }\end{array}$ \\
\hline $\begin{array}{l}\text { Careya arborea } \\
\text { (Barringtoniaceae) }\end{array}$ & $\sqrt{ }$ & & - & & $\begin{array}{l}\text { Medium tree } \\
15-20 \mathrm{~m} \text { tall }\end{array}$ & $\begin{array}{l}\text { Fibrous bark: body } \\
\text { swellings }\end{array}$ & $\begin{array}{l}\text { Timber: construction } \\
\text { furniture and agricultural } \\
\text { implements. }\end{array}$ \\
\hline $\begin{array}{l}\text { Coffea arabica } \\
\text { (Rubiaceae) }\end{array}$ & - & $\sqrt{ }$ & $\sqrt{ }$ & & $\begin{array}{l}\text { shrub or small } \\
\text { tree, } 5 \mathrm{~m} \text { tall }\end{array}$ & \begin{tabular}{|l|} 
Dried ripe seeds are \\
stimulant, on central \\
nervous system, kidneys, \\
heart, and muscles
\end{tabular} & $\begin{array}{l}\text { Coffee is source of } \\
\text { caffeine. }\end{array}$ \\
\hline \begin{tabular}{|l}
$\begin{array}{l}\text { Cullenia exallirata } \\
\text { (Bombaceae) }\end{array}$ \\
\end{tabular} & $\sqrt{ }$ & $\sqrt{ }$ & - & & Large tree $40 \mathrm{~m}$ & & $\begin{array}{l}\text { Nectar: food source for } \\
\text { endemic animals }\end{array}$ \\
\hline \begin{tabular}{|l} 
Dichapetalum \\
gelonioides \\
(Dichapetalaceae) \\
\end{tabular} & - & $\sqrt{ }$ & $\sqrt{ }$ & & $\begin{array}{l}\text { Large } \\
\text { trees,20-25 m } \\
\text { tall } \\
\end{array}$ & & $\begin{array}{l}\text { Fruit:Fruit:Feed for } \\
\text { frugivorous bats and birds }\end{array}$ \\
\hline \begin{tabular}{|l|} 
Diospyros foliolosa \\
(Ebenaceae)
\end{tabular} & - & $\sqrt{ }$ & - & & Trees $12 \mathrm{~m}$ tall. & $\begin{array}{l}\text { Bark used to treat } \\
\text { diarrohea and dyspesia. }\end{array}$ & $\begin{array}{l}\text { Fruit: Feed for } \\
\text { frugivorous bats and birds }\end{array}$ \\
\hline $\begin{array}{l}\text { Diospyros sylvatica } \\
\text { (Ebenaceae) }\end{array}$ & - & $\sqrt{ }$ & - & & $\begin{array}{l}\text { Trees up to } 35 \mathrm{~m} \\
\text { tall. }\end{array}$ & & $\begin{array}{l}\text { Fruit: Feed for } \\
\text { frugivorous bats and birds }\end{array}$ \\
\hline \begin{tabular}{|l} 
Name of Core Plant \\
Species
\end{tabular} & Flower & Fruit & Seedlings & Location & Tree height (m) & Medicinal importance & Ecological importance \\
\hline \begin{tabular}{|l|}
$\begin{array}{l}\text { Drypetes roxburghii } \\
\text { (Euphorbiaceae) }\end{array}$ \\
\end{tabular} & - & $\sqrt{ }$ & - & & $\begin{array}{l}\begin{array}{l}\text { Trees up to } 15 \mathrm{~m} \\
\text { tall }\end{array} \\
\end{array}$ & $\begin{array}{l}\text { Leaves and fruits: } \\
\text { Medicine for rheumatism. }\end{array}$ & $\begin{array}{l}\text { Fruit: Feed for } \\
\text { frugivorous bats and birds }\end{array}$ \\
\hline \begin{tabular}{|l} 
Elaeocarpus \\
serratus L. \\
(Eleocarpaceae) \\
\end{tabular} & - & $\sqrt{ }$ & - & & $\begin{array}{l}\text { Trees up to } 18 \mathrm{~m} \\
\text { tall. }\end{array}$ & $\begin{array}{l}\text { Leaves rheumatism. } \\
\text { Fruits: dysentery and } \\
\text { diarrhea. }\end{array}$ & $\begin{array}{l}\text { Fruit: Feed for } \\
\text { frugivorous bats and birds }\end{array}$ \\
\hline $\begin{array}{l}\text { Eleaocarpus } \\
\text { tuberculatus } \\
\text { (Elaeocarpaceae) }\end{array}$ & - & $\sqrt{ }$ & - & & $\begin{array}{l}\text { Tall trees } 40 \mathrm{~m} \\
\text { tall. }\end{array}$ & $\begin{array}{l}\text { Bark: haematemes is } \\
\text { biliousness and } \\
\text { indigestion. Fruits used in } \\
\text { typhoid rheumatism and } \\
\text { epilepsy. }\end{array}$ & $\begin{array}{l}\text { Fruit: Feed for } \\
\text { frugivorous bats and birds }\end{array}$ \\
\hline \begin{tabular}{|l|}
$\begin{array}{l}\text { Eleocarpus munronii } \\
\text { (Elaeocarpaceae) }\end{array}$ \\
\end{tabular} & - & $\sqrt{ }$ & - & & $\begin{array}{l}\text { Trees up to } 15 \mathrm{~m} \\
\text { tall }\end{array}$ & & $\begin{array}{l}\text { Fruit: Feed for } \\
\text { frugivorous bats and birds }\end{array}$ \\
\hline $\begin{array}{l}\text { Ensete superbum } \\
\text { (Musaceae) }\end{array}$ & $\sqrt{ }$ & & - & & $\begin{array}{l}\text { small tree } 12 \mathrm{ft} \\
\text { in height }\end{array}$ & Fruit: To treat diabetics & $\begin{array}{l}\text { The flower of the plant is } \\
\text { visited by bats to take } \\
\text { nectar. }\end{array}$ \\
\hline \begin{tabular}{|l} 
Eriodendron \\
pentandrum \\
(Bombaceae) \\
\end{tabular} & $\sqrt{ }$ & & - & & $\begin{array}{l}\text { Trees, usually } \\
\text { large }\end{array}$ & & cotton taken from the fruit \\
\hline $\begin{array}{l}\text { Erythrina indica } \\
\text { (Papilionaceae) }\end{array}$ & $\sqrt{ }$ & & - & & $\begin{array}{l}\text { medium-sized } \\
\text { tree } 15-20 \mathrm{~m} \text { tall }\end{array}$ & $\begin{array}{l}\text { Leaves: laxative, apllied } \\
\text { externally on venereal } \\
\text { buboes. }\end{array}$ & $\begin{array}{l}\text { Wood: construct floats. } \\
\text { The flower becomes food } \\
\text { for bat species. }\end{array}$ \\
\hline \begin{tabular}{|l} 
Erythroxylum \\
monogynum \\
(Erythroxylaceae) \\
\end{tabular} & - & $\sqrt{ }$ & - & & $\begin{array}{l}\text { small tree } \\
15-20 \mathrm{~m} \text { tall }\end{array}$ & $\begin{array}{l}\text { fruit: To treat dyspepsia } \\
\text { and fever. }\end{array}$ & $\begin{array}{l}\text { The wood is used as a } \\
\text { substitute for Sandal. }\end{array}$ \\
\hline $\begin{array}{l}\text { Ficus asperima } \\
\text { (Moraceae) }\end{array}$ & - & $\sqrt{ }$ & $\mid-$ & & tree $18 \mathrm{~m}$ tall & & Polish for furnitures. \\
\hline
\end{tabular}


Table 1 to be continued

\begin{tabular}{|c|c|c|c|c|c|c|c|}
\hline $\begin{array}{l}\text { Name of Core Plant } \\
\text { Species }\end{array}$ & Flower & Fruit & Seedlings & Location & Tree height (m) & Medicinal importance & Ecological importance \\
\hline \begin{tabular}{|l}
$\begin{array}{l}\text { Ficus beddomei } \\
\text { (Moraceae) }\end{array}$ \\
\end{tabular} & - & $\sqrt{ }$ & - & & tree $35-40 \mathrm{~m}$ tall & & Silk production \\
\hline \begin{tabular}{|l}
$\begin{array}{l}\text { Ficus callosa } \\
\text { (Moraceae) }\end{array}$ \\
\end{tabular} & - & $\sqrt{ }$ & - & & Trees: $25 \mathrm{~m}$ tall. & & $\begin{array}{l}\text { Wood: Furniture and boat } \\
\text { making. }\end{array}$ \\
\hline \begin{tabular}{|l}
$\begin{array}{l}\text { Ficus guttata } \\
\text { (Moraceae) }\end{array}$ \\
\end{tabular} & - & $\sqrt{ }$ & - & & $\begin{array}{l}\text { Trees large } \\
30-35 \mathrm{~m} \text { tall }\end{array}$ & & $\begin{array}{l}\text { Fruit: Feed for endemic } \\
\text { frugivorous bats and birds }\end{array}$ \\
\hline \begin{tabular}{|l}
$\begin{array}{l}\text { Ficus racemosa } \\
\text { (Moraceae) }\end{array}$ \\
\end{tabular} & - & $\sqrt{ }$ & - & & \begin{tabular}{|l|} 
Trees large \\
$30-35 \mathrm{~m}$ tall \\
\end{tabular} & $\begin{array}{l}\text { Fruit extract: To treat } \\
\text { diabetes, leucoderma and }\end{array}$ & $\begin{array}{l}\text { Edible fruir for animals } \\
\text { and bird. }\end{array}$ \\
\hline \begin{tabular}{|l|}
$\begin{array}{l}\text { Ficus retusa } \\
\text { (Moraceae) }\end{array}$ \\
\end{tabular} & - & $\sqrt{ }$ & - & & $\begin{array}{l}\text { Huge tree } 60 \text { to } \\
70 \mathrm{ft} . t a l l\end{array}$ & $\begin{array}{l}\text { Bark: To treat colic } \\
\text { disorders }\end{array}$ & $\begin{array}{l}\text { Fruit: Feed for endemic } \\
\text { frugivorous bats and birds }\end{array}$ \\
\hline $\begin{array}{l}\text { Madhuca longifolia } \\
\text { (Sapotaceae) }\end{array}$ & - & $\sqrt{ }$ & - & & $\begin{array}{l}\text { Trees, } 20 \text { meters } \\
\text { in height }\end{array}$ & $\begin{array}{l}\text { The flowers, seeds and } \\
\text { seed oil have great } \\
\text { medicinal value. }\end{array}$ & $\begin{array}{l}\text { Fruit: Feed for } \\
\text { frugivorous bats and birds }\end{array}$ \\
\hline $\begin{array}{l}\text { Mangifera indica } \\
\text { (Anacardiaceae) }\end{array}$ & - & - & $\sqrt{ }$ & & $\begin{array}{l}\text { Beautiful, large } \\
\text { tropical tree to } \\
50-80 \mathrm{ft}\end{array}$ & $\begin{array}{l}\text { Bark: To treat uterine } \\
\text { haemorrhage, Asthma }\end{array}$ & $\begin{array}{l}\text { Fruit: Feed for } \\
\text { frugivorous bats and birds }\end{array}$ \\
\hline $\begin{array}{l}\text { Musua ferrea } \\
\text { (Musaceae) }\end{array}$ & - & $\sqrt{ }$ & & & $\begin{array}{l}\text { small evergreen } \\
\text { tree } 15-20 \mathrm{~m} \text { tall }\end{array}$ & $\begin{array}{l}\text { This oil has antibacterial, } \\
\text { antifungal and } \\
\text { anthelmintic }\end{array}$ & $\begin{array}{l}\text { Timber: railroad ties and } \\
\text { heavy structural. }\end{array}$ \\
\hline \begin{tabular}{|l}
$\begin{array}{l}\text { Nephelium longana } \\
\text { (Sapindaceae) }\end{array}$ \\
\end{tabular} & - & $\sqrt{ }$ & $\sqrt{ }$ & & tree $45 \mathrm{~m}$ tall & & $\begin{array}{l}\text { Fruit: Feed for } \\
\text { frugivorous bats and birds }\end{array}$ \\
\hline $\begin{array}{l}\text { Palaquium } \\
\text { ellipticum } \\
\text { (Sapotaceae) } \\
\end{array}$ & - & $\sqrt{ }$ & $\sqrt{ }$ & & tree $35 \mathrm{~m}$ tall & & $\begin{array}{l}\text { latex is bioinert, resilient, } \\
\text { and is a good electrical } \\
\text { insulator }\end{array}$ \\
\hline $\begin{array}{l}\text { Todalia asiatica(L.) } \\
\text { Lam (Rutaceae) }\end{array}$ & - & $\sqrt{ }$ & - & & height of $10 \mathrm{~m}$ & $\begin{array}{l}\text { Fruit: cough remedy, to } \\
\text { treat indigestion and } \\
\text { influenza }\end{array}$ & $\begin{array}{l}\text { Fruit: Feed for } \\
\text { frugivorous bats and birds }\end{array}$ \\
\hline $\begin{array}{l}\text { Name of Core Plant } \\
\text { Species }\end{array}$ & Flower & Fruit & Seedlings & Location & Tree height (m) & Medicinal importance & Ecological importance \\
\hline \begin{tabular}{|l} 
Strychnos \\
cinnnamomifolia or \\
Strychnos minor \\
(Loganiaceae) \\
\end{tabular} & - & $\sqrt{ }$ & - & & & $\begin{array}{l}\text { Wood: Tonic in dyspepsia } \\
\text { and malarial affections. }\end{array}$ & $\begin{array}{l}\text { Fruit: Feed for } \\
\text { frugivorous bats and birds }\end{array}$ \\
\hline $\begin{array}{l}\text { Syzygium cumini } \\
\text { (Myrtaceae) }\end{array}$ & - & $\sqrt{ }$ & $\sqrt{ }$ & & 50 to $100 \mathrm{ft}$. tall & $\begin{array}{l}\text { Bark: anemia, diabetes. } \\
\text { Fruit dysentery. Leaf } \\
\text { juice: antibacterial. }\end{array}$ & $\begin{array}{l}\text { Wood: durable in water, } \\
\text { resistant to termites, } \\
\text { furnitre plywood, }\end{array}$ \\
\hline $\begin{array}{l}\text { Syzygium jambos } \\
\text { (Myrtaceae) }\end{array}$ & - & $\sqrt{ }$ & - & & $10-30 \mathrm{~m}$ tall & $\begin{array}{l}\text { Bark used as gargles and } \\
\text { mouth washes, Seeds used } \\
\text { in diabetes }\end{array}$ & Fruit extract: rose water. \\
\hline \begin{tabular}{|l} 
Syzygium \\
mundagam \\
(Myrtaceae) \\
\end{tabular} & $\sqrt{ }$ & $\sqrt{ }$ & - & & Trees 20 m tall. & $\begin{array}{l}\text { Essential oils with a high } \\
\text { vitamin }\end{array}$ & $\begin{array}{l}\text { Fruit: Feed for } \\
\text { frugivorous bats and birds }\end{array}$ \\
\hline $\begin{array}{l}\text { Syzygium } \\
\text { tamilnadensis } \\
\text { (Myrtaceae) } \\
\end{array}$ & - & - & $\sqrt{ }$ & & Trees $25 \mathrm{~m}$ tall & $\begin{array}{l}\text { Essential oils with a high } \\
\text { vitamin content }\end{array}$ & $\begin{array}{l}\text { Fruits feed for bats and } \\
\text { primates .Flower yield } \\
\text { nectar for birds and bats. }\end{array}$ \\
\hline
\end{tabular}

\begin{tabular}{|l|l|}
\hline Endemic : South Western Ghats & Theni \\
\hline Southern Western Ghats & Tirunelveli \\
\hline Western Ghats & Theni and Tirunelveli \\
\hline
\end{tabular}

aids the forests to establish a balanced ecosystem with biodiversity.

Food selection and the 'bat-plant' interaction helped to assess the bat's propagating impact on the ever green forests of Western Ghats. Latidens is a disperser of plant species in which natural germination beneath or around the parent tree is not possible (Vanitharani 2005). The bat selects fruits with more or less dull cryptic coloration like green, brown or purplish black and were displayed clear off the foliage. Generally 


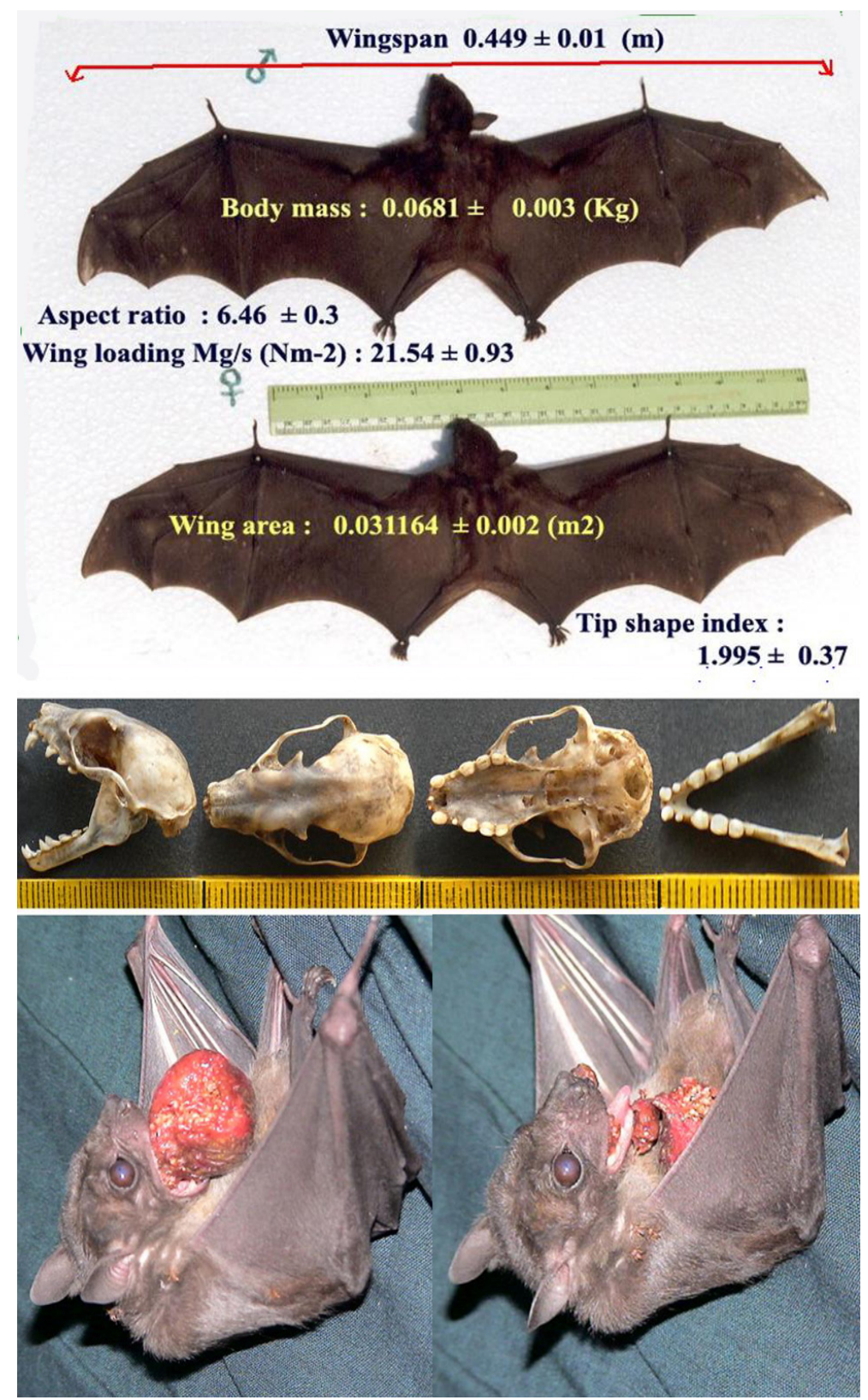

Plate 4 Skull, dental and wing morphology of $\boldsymbol{L}$. salimalii helps the feeding and foraging performance. Latidens salimalii eating Ficus racemosa fruit while processing. the fruit is held in its wing pouch.

Table 2a Cranial measurements of L. salimalii.

\begin{tabular}{lc}
\hline Expansion of the abbreviations & \\
\hline SIZE: Sum of the natural logs of condylo-canine length, zygomatic breadth and temporal height & $8.9(\log )$ \\
GTL: Greatest skull length & $32.4(\mathrm{~mm})$ \\
CCL: Condylo-canine length & $31.1(\mathrm{~mm})$ \\
BB: Breadth of brain & $14.0(\mathrm{~mm})$ \\
ZB: Zygomatic breadth & $20.1(\mathrm{~mm})$ \\
MSW: Minimum skull width & $7.5(\mathrm{~mm})$ \\
PSW: Posterior skull width & $14.3(\mathrm{~mm})$ \\
TH: Temporal height & $11.6(\mathrm{~mm})$ \\
UC: Upper canine. & $3.3(\mathrm{~mm})$ \\
\hline
\end{tabular}


Table 2b Dental measurements of Latidens salimalii.

\begin{tabular}{|c|c|c|c|c|c|c|c|c|c|c|c|c|c|}
\hline \multicolumn{13}{|c|}{ Dental measurements (mm) } & \multirow{3}{*}{$\begin{array}{l}\text { Dental formula } \\
\text { i-incisor; c-canine; } \\
\text { p-premolar; m-molar }\end{array}$} \\
\hline \multicolumn{4}{|c|}{ Upper jaw } & \multicolumn{9}{|c|}{ Lower jaw } & \\
\hline$\overline{\mathrm{PC}}$ & $\mathrm{PM}_{1}$ & $\mathrm{PM}_{3}$ & $\mathrm{CM}^{\mathrm{n}}$ & $\mathrm{CM}_{\mathrm{n}}$ & $\mathrm{M}$ & DD & DT & $\mathrm{CPH}$ & $\mathrm{CM}$ & Conl & $\mathrm{CC}$ & Concor & \\
\hline 5.80 & 8.4 & 10.0 & 10.90 & 12.2 & 25.15 & 2.80 & 1.30 & 10.60 & 12.54 & 5.45 & 24.3 & 7.2 & $\begin{array}{l}-2-1-2341-- \\
\mathrm{i}----\mathrm{c}---\mathrm{pm}----\mathrm{m}---=26 \\
-2-1-23412-\end{array}$ \\
\hline
\end{tabular}

Expansion of the abbreviations used in the table is given in parenthesis.

PC: Anterior palatal width; PM1: Palatal width from M1; PM3: Posterior palatal width;

CMn: Maxillary tooth row; CMn: Mandibular tooth row; M: Mandible length;

DD: Dentary depth; DT: Dentary thickness; CPH: Coronoid process height;

CM: Condyle to lower molar toothrow; Conl: Condyle length; CC: Condyle to canine;

$\mathrm{CC}$ : Condyle to canine; Concor: Condyle to coronoid process.

Table 3a Measurements of wing morphology of Latidens salimalii.

\begin{tabular}{|c|c|c|c|c|c|c|c|c|c|c|c|c|c|c|}
\hline \multirow[b]{2}{*}{$\mathrm{N}$} & \multirow[b]{2}{*}{ Sex } & \multirow{2}{*}{$\begin{array}{l}\text { Wing } \\
\text { span } \\
(\mathrm{mm})\end{array}$} & \multicolumn{12}{|c|}{ Fingers $(\mathrm{mm})$} \\
\hline & & & Thumb & $\begin{array}{l}2 \text { meta } \\
\text { carpal }\end{array}$ & $\begin{array}{l}3 \text { meta } \\
\text { carpal }\end{array}$ & $\begin{array}{l}\text { 4meta } \\
\text { carpal }\end{array}$ & $\begin{array}{l}\text { 5meta } \\
\text { carpal }\end{array}$ & $\begin{array}{l}1 \mathrm{ph} \\
2 \mathrm{mt}\end{array}$ & $1 \mathrm{ph} 3 \mathrm{mt}$ & $2 \mathrm{ph} 3 \mathrm{mt}$ & $1 \mathrm{ph} 4 \mathrm{mt}$ & $2 \mathrm{ph} 4 \mathrm{mt}$ & $1 \mathrm{ph} 5 \mathrm{mt}$ & $2 \mathrm{ph} 5 \mathrm{mt}$ \\
\hline 5 & $\widehat{\delta}$ & $\begin{array}{l}479 \pm \\
6.52\end{array}$ & $\begin{array}{l}19 \pm \\
1.581\end{array}$ & $\begin{array}{l}26.4 \pm \\
1.949\end{array}$ & $\begin{array}{l}44.6 \pm \\
0.894\end{array}$ & $\begin{array}{l}44.6 \pm \\
0.894\end{array}$ & $\begin{array}{l}43.4 \pm \\
0.894\end{array}$ & $\begin{array}{l}14.2 \pm \\
1.304\end{array}$ & $\begin{array}{l}32.2 \pm \\
1.304\end{array}$ & $\begin{array}{l}41.8 \pm \\
0.447\end{array}$ & $\begin{array}{l}25.2 \pm \\
1.643\end{array}$ & $\begin{array}{l}28.2 \pm \\
0.837\end{array}$ & $\begin{array}{l}21.8 \pm \\
0.837\end{array}$ & $\begin{array}{l}21.8 \pm \\
1.095\end{array}$ \\
\hline 5 & q & $\begin{array}{l}485.6 \pm \\
10.213\end{array}$ & $\begin{array}{l}21.4 \pm \\
2.608\end{array}$ & $\begin{array}{l}24.4 \pm \\
2.51\end{array}$ & $\begin{array}{l}47.4 \pm \\
1.517\end{array}$ & $\begin{array}{l}45.4 \pm \\
1.342\end{array}$ & $\begin{array}{l}44.8 \pm \\
1.095\end{array}$ & $\begin{array}{l}13.4 \pm \\
1.673\end{array}$ & $\begin{array}{l}32.6 \pm \\
1.673\end{array}$ & $\begin{array}{l}45.8 \pm \\
2.168\end{array}$ & $\begin{array}{l}27 \pm \\
1.225\end{array}$ & $\begin{array}{l}29.4 \pm \\
0.894\end{array}$ & $\begin{array}{l}21.4 \pm \\
0.894\end{array}$ & $\begin{array}{l}21.8 \pm \\
0.447\end{array}$ \\
\hline
\end{tabular}

Value: Mean \pm Standard deviation. N: number of individuals. Abbreviations used to denote fingers.

1ph 2mt - 1 Phalanx of 2 metacarpal; 1ph 3mt - 1 Phalanx of 3 metacarpal; 2ph 3mt - 2 Phalanx of 3 metacarpal;

1ph $4 \mathrm{mt}$ - 1 Phalanx of 4 metacarpal; 2ph 4mt - 2 Phalanx of 4 metacarpal; 1ph 5mt - 1 Phalanx of 5 metacarpal;

2ph 5mt - 2 Phalanx of 5 metacarpal.

Table 3b Body mass and aerodynamic wing dimensions of Latidens salimalii.

\begin{tabular}{|c|c|c|c|c|c|c|c|c|c|}
\hline $\begin{array}{l}\text { Body mass } \\
\mathrm{M}(\mathrm{Kg})\end{array}$ & $\begin{array}{l}\text { Wingspan } \\
\text { B (m) }\end{array}$ & $\begin{array}{l}\text { Wing area } \\
\mathrm{S}\left(\mathrm{m}^{2}\right)\end{array}$ & $\begin{array}{l}\text { Aspect } \\
\text { ratio (A) }\end{array}$ & $\begin{array}{l}\text { Wing } \\
\text { loading } \\
(\mathrm{Mg} / \mathrm{S} \\
\left(\mathrm{NM}^{-2}\right)\end{array}$ & $\begin{array}{l}\text { Tip length } \\
\text { ratio } \mathrm{Tl}\end{array}$ & $\begin{array}{l}\text { Tip area } \\
\text { ratio Ts }\end{array}$ & $\begin{array}{l}\text { Tip shape } \\
\text { index I }\end{array}$ & $\begin{array}{l}\text { Wing loading } \\
\text { index } \mathrm{M}^{2 / 3} / \mathrm{S}\end{array}$ & $\begin{array}{l}\text { No. of } \\
\text { individuals }\end{array}$ \\
\hline $0.0681 \pm 0.00$ & $0.449 \pm$ & $0.0312 \pm 0.04$ & $6.46 \pm 0.4$ & $21.5 \pm 1.5$ & $1.02 \pm 0.02$ & $0.67 \pm 0.01$ & $2 \pm 0.03$ & $5.35 \pm 0.036$ & 5 \\
\hline
\end{tabular}

prefer fresh clusters of ripened fruits with matured and good vigor seeds and never scavenge on fallen fruits. The morphological features like wide mouth with strong skull and dentition fecilitates a grip and carry fruits for long distance flight (Freeman 1988). The aerodynamickally adapted wings help maneuverable flight during foraging (Norberg and Rayner 1987). Thus the bats do food selection in the canopy, sub canopy levels and within the cluttered forest cover. The listed bat preferred core plant species show a wide range of seeds, with varied fruit size and seed number. Some are soft and hard-fleshed, protected (by edible rind) and unprotected.Varieties of food remains from single nights foraging indicates $L$. salimalii has wide foraging patterns, home-range movements and visiting more variety of plant species to get balanced diet by which creating a wide dispersal.

\subsection{Feeding Roost and Food Processing}

The fruit bats handle the fruits and flowers very gently without causing any damage to the flowers and seeds. L. salimalii selects open or wide mouthed cave roof or tall trees with horizontal branches as feeding roost. To obviate the need to travel too long distances while carrying fruits they select a feeding roost closer to the foraging area, usually within the radius of $1 \mathrm{~km}$ from the fruiting tree. Occasionally fruits were carried much further.

Food processing in the feeding roost involve several steps depending on fruit size and morphology. Larger 


\section{Latidens Salimalii (Endemic, Endanger Fruit Bat) A Reliable Propagator of Endemic Trees of Southern Western Ghats}

Table 4a Location of bat feeding roost in Agasthiyamali Biospher Reserve and the available seeds and seedlings.

\begin{tabular}{|c|c|c|c|c|}
\hline Sl. No & $\begin{array}{l}\text { Seeds and Seedlings } \\
\text { available in the feeding } \\
\text { roosting }\end{array}$ & Family & Study area & GPS Location \\
\hline 1 & Mangifera indica & Anacardiaceae & Shengaltheri & 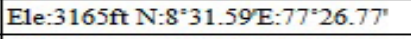 \\
\hline 2 & Careya arborea & Barringtoniaceae & Shengaltheri & Ele:3112ft N:8 $31.97 \mathrm{E}: 77^{\circ} 27.26^{\prime \prime}$ \\
\hline 3 & Cullenia exallirata & Bombaceae & $\begin{array}{l}\text { Kudhiraivetti, Valaiyar, } \\
\text { Shengaltheri }\end{array}$ & 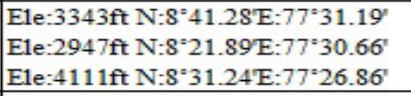 \\
\hline 4 & Eriodendron penandrum & Bombaceae & Therkumalai & Ele:1890ft N:843.08E:77"41.85" \\
\hline 5 & Dichapetalum jelonioides & Dichapetalaceae & Therkumalai & $\begin{array}{l}\text { Ele:2412ft N:8 } 53.88^{\circ}: 77^{\circ} 15.28^{\prime \prime} \\
\text { Ele:2442ft N:8 } 8^{\circ} 53.08^{\prime} \mathrm{E}: 77^{\circ} 41.85^{\prime \prime} \\
\text { Ele:3174ft N:8"53.99E: } 77^{\circ} 15.35^{\prime \prime}\end{array}$ \\
\hline 6 & Diospyros foliolosa & Ebenaceae & Therkumalai & Ele:2442ft N:8 $53.08 \mathrm{E}: 77^{\circ} 41.85^{\prime \prime}$ \\
\hline 7 & Diospyros sylvatica & Ebenaceae & Shengaltheri & 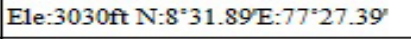 \\
\hline 8 & Eleocarpus munronit & Elaeocarpaceae & Shengaltheri & Ele:3541ft N:8 $31.59 \mathrm{E}: 77^{\circ} 26.78^{\prime \prime}$ \\
\hline 9 & Elaeocarpus serratus & Elaeocarpaceae & $\begin{array}{l}\text { Sivagiri (Kallakadai } \\
\text { estate), Poongulam, } \\
\text { Shengaltheri, } \\
\text { Therkumalai }\end{array}$ & 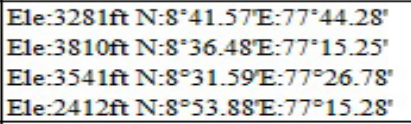 \\
\hline 10 & Eleaocarpus tuberculatus & Elaeocarpaceae & $\begin{array}{l}\text { Therkumalai, } \\
\text { Shengaltheri }\end{array}$ & 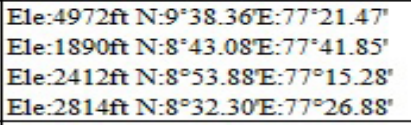 \\
\hline 11 & Erythroxylum monogynum & Erythroxylaceae & Shengaltheri & Ele:3542ft N:8 $31.59 \mathrm{E}: 77^{\circ} 26.7 \mathrm{~S}^{\prime \prime}$ \\
\hline 12 & Ficus asperima & Moraceae & $\begin{array}{l}\text { Thalayanai (Sivagiri), } \\
\text { Sivagiri (Kallakadai } \\
\text { estate) }\end{array}$ & 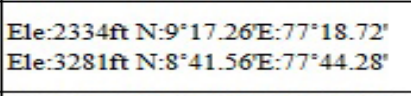 \\
\hline 13 & Ficus beddomei & Moraceae & Therkumalai & $\begin{array}{l}\text { Ele:2412ft N:8 } 8^{\circ} 53.88^{\prime} \mathrm{E}: 77^{\circ} 15.28^{\prime \prime} \\
\text { Ele:2040ft N:8 } 43.08^{\circ} \mathrm{E}: 77^{\circ} 41.85^{\prime \prime}\end{array}$ \\
\hline 14 & Ficus callosa & Moraceae & Shengaltheri & $\begin{array}{l}\text { Ele:3541ft N:8 } 8^{\circ} 31.59 \mathrm{E}: 77^{\circ} 26.78^{\prime \prime} \\
\text { Ele:3429ft N:8 } 43.08 \mathrm{E}: 77^{\circ} 41.85^{\prime \prime}\end{array}$ \\
\hline 15 & $\begin{array}{l}\text { Ficus guttata (Spat outs on } \\
\text { rocks }\end{array}$ & Moraceae & Shengaltheri & $\begin{array}{l}\text { Ele:3595ft N:8 } 8^{\circ} 31.42 \mathrm{E}: 77^{\circ} 26.36^{\prime \prime} \\
\text { Ele:2814ft N:8 } 32.03 \mathrm{E}: 77^{\circ} 26.88^{\prime \prime}\end{array}$ \\
\hline 16 & Eleocarpus munronii & Elaeocarpaceae & Shengaltheri & Ele:3541ft N:8 $31.59 \mathrm{E}: 77^{\circ} 26.78^{\prime \prime}$ \\
\hline 17 & Ficus racemosa & Moraceae & \begin{tabular}{|l|} 
Servalaru, \\
Naraikadu \\
\end{tabular} & $\begin{array}{l}\text { Ele: } 757 \mathrm{ft} \mathrm{N}: 8^{\circ} 41.17^{\prime} \mathrm{E}: 77^{\circ} 18.77^{\prime \prime} \\
\text { Ele: } 1185 \mathrm{ft} \mathrm{N}: 8^{\circ} 27.16 \mathrm{E}: 77^{\circ} 30.56^{\prime \prime}\end{array}$ \\
\hline 18 & Ficus retusa. & Moraceae & Shengaltheri & Ele:2814ft N:8 $32.03 \mathrm{E}: 77^{\circ} 26.87^{\prime \prime}$ \\
\hline 19 & Ensete superbum & Musaceae & Ingikuli & Ele: $1947 \mathrm{ft} \mathrm{N}: \mathrm{S}^{\circ} 37.38^{\prime} \mathrm{E}: 77^{\circ} 16.78^{\circ}$ \\
\hline 20 & Musua ferrea & Musaceae & Therkumalai & Ele:2412ft N:8 $53.88 \mathrm{E}: 77^{\circ} 15.28^{\prime \prime}$ \\
\hline 21 & Syzygium cumini & Myrtaceae & Shengaltheri & 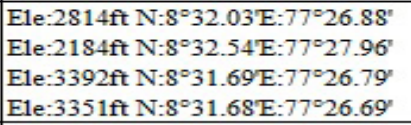 \\
\hline 22 & Syzygium jambos & Myrtaceae & Therkumalai & 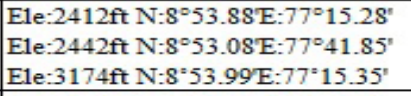 \\
\hline 23 & Syzygium mundagam & Myrtaceae & $\begin{array}{l}\text { Shengaltheri, } \\
\text { Therkumalai }\end{array}$ & 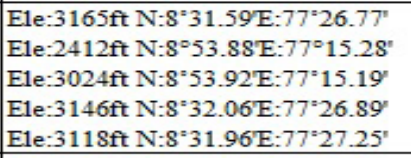 \\
\hline 24 & Erythrina indica & Papilionaceae & $\begin{array}{l}\text { Sivagiri (Kallakadai } \\
\text { estate) }\end{array}$ & 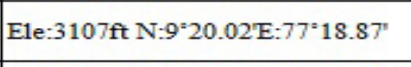 \\
\hline 25 & Achronychia pedunculata & Rutaceae & Shengaltheri & Ele:2911ft N:8 $32.18 \mathrm{E}: 77^{\circ} 27.47^{\prime \prime}$ \\
\hline 26 & Atalantia monophylla & Rutaceae & Therkumalai & Ele: $1194 \mathrm{ft} N: 8^{2} 31.94 \mathrm{E}: 77^{\circ} 26.89$ \\
\hline 27 & Madhuca longifolia & Sapotaceae & Shengaltheri & \begin{tabular}{|l|} 
Ele:2852ft N:8 $88^{\circ} 1.77^{\prime} \mathrm{E}: 77^{\circ} 26.82^{\prime \prime}$ \\
Ele:1918ft N:8 $32.54 \mathrm{E}: 77^{\circ} 27.98^{\prime \prime}$
\end{tabular} \\
\hline 28 & Palaquium ellipticum & Sapotaceae & $\begin{array}{l}\text { Kudhiraivetti, } \\
\text { Shengaltheri }\end{array}$ & 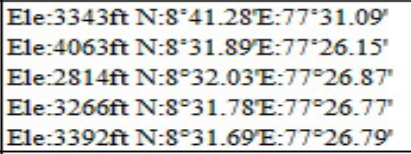 \\
\hline 29 & Nephelium longana & Sapindaceae & Therkumalai & Ele:2412ft N:8 $53.88 \mathrm{E}: 77^{\circ} 15.28^{\prime \prime}$ \\
\hline 30 & Myristica dactyloides & Myristicaceae & Mahndragiri forest & Ele:2657ft N:8"26.26'E:77"24.56' \\
\hline 31 & Psychotriasp & Rubiaceae & Mahndragiri forest & 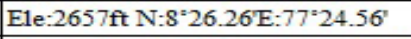 \\
\hline 32 & Tarena asiatica & Rubiaceae & Mahndragiri forest & Ele: $2657 \mathrm{ft} \mathrm{N}: 08^{2} 26.26 \mathrm{E}: 77^{\prime} 24.56^{\prime}$ \\
\hline 33 & Dimocarpus longan & Sapindaceae & Mahndragiri forest & Ele:2657ft N:08 $26.26^{\prime} \mathrm{E}: 77^{\prime} 24.56^{\prime \prime}$ \\
\hline 34 & Palaquium ellipticum & Sapotaceae & Mahndragiri forest & Ele:2657ft N:08 $26.26 \mathrm{E}: 77^{\prime} 24.56^{\prime}$ \\
\hline
\end{tabular}


Table 4b Location of bat feeding roost in High Wavey Mountains and the available seeds and seedlings.

\begin{tabular}{|c|c|c|c|}
\hline SI. No & $\begin{array}{l}\text { Seeds and Seedlings } \\
\text { available in the feeding roost }\end{array}$ & Area of collection & GPS Location \\
\hline 1 & $\begin{array}{l}\text { Cullenia exallirata } \\
\text { (Bombaceae) }\end{array}$ & Highwavys & Elev: 4987ft N:09?39.501' E:077?22.010' \\
\hline 2 & $\begin{array}{l}\text { Bhesa indica (Bedd.) Ding } \\
\text { (Celastraceae) }\end{array}$ & $\begin{array}{l}\text { Pudhukadu, } \\
\text { Kardana cave }\end{array}$ & $\begin{array}{l}\text { Elev: } 5207 \mathrm{ft} \mathrm{N}: 09 ? 38.264 ' \text { E:077?21.847' } \\
\text { Elev: 4994ft N: 09?38.391' E:077?22.034' } \\
\text { Elev: } 5009 \mathrm{ft} \mathrm{N}: 09 ? 38.380^{\prime} \mathrm{E}: 077 ? 22.011^{\prime} \\
\text { Elev: } 3709 \mathrm{ft} \mathrm{N}: 09 ? 41.610^{\prime} \mathrm{E}: 077 ? 24.232^{\prime} \\
\text { Elev: } 4748 \mathrm{ft} \mathrm{N}: 09 ? 39.709^{\prime} \mathrm{E}: 077 ? 21.728^{\prime}\end{array}$ \\
\hline 3 & Mesua ferrea (Clusiaceae) & Venniyar & Elev: 4746ft N:09?38.259' E:077?21.264' \\
\hline 4 & $\begin{array}{l}\text { Dichapetalum gelonioides } \\
\text { (Roxb)Engl (Dichapetalaceae) }\end{array}$ & $\begin{array}{l}\text { Pudhukadu, } \\
\text { Kardana cave, } \\
\text { Venniyar }\end{array}$ & $\begin{array}{l}\text { Elev: } 5009 \mathrm{ft} \mathrm{N}: 09 ? 38.380^{\prime} \mathrm{E}: 077 ? 22.011^{\prime} \\
\text { Elev: } 3709 \mathrm{ft} \mathrm{N}: 09 ? 41.610^{\prime} \mathrm{E}: 077 ? 24.232^{\prime} \\
\text { Elev: 4749ft N: 09?37.059' E:077?19.438' }\end{array}$ \\
\hline 5 & $\begin{array}{l}\text { Diospyros foliolosa } \\
\text { (Ebenaceae) }\end{array}$ & Highwavys & Elev: 4830ft N:09?38.128' E:077?21.498' \\
\hline 6 & $\begin{array}{l}\text { Eleocarpus munronii } \\
\text { (Elaeocarpaceae) }\end{array}$ & Kardana estate & Elev: $3608 \mathrm{ft}, \mathrm{N}: 09 ? 41.626$ ' E:077?24.104' \\
\hline 7 & $\begin{array}{l}\text { Eleaocarpus tuberculatus } \\
\text { (Elaeocarpaceae) }\end{array}$ & Highwavys & $\begin{array}{l}\text { Elev: } 4972 \mathrm{ft} \mathrm{N}: 09 ? 38.365^{\prime} \text { E: } 077 ? 21.474^{\prime} \\
\text { Elev: } 1890 \mathrm{ft} \mathrm{N}: 08 ? 43.083^{\prime} \text { E: } 077 ? 41.854^{\prime} \\
\text { Elev: } 2412 \mathrm{ft} \mathrm{N}: 08^{\circ} 53.838^{\prime} \text { E: } 077^{\circ} 15.248^{\prime}\end{array}$ \\
\hline 8 & $\begin{array}{l}\text { Elaeocarpus serratus } L . \\
\text { (Eleocarpaceae) }\end{array}$ & Kardana cave & Elev: 3709ft N: 09?41.610' E:077?24.232' \\
\hline 9 & $\begin{array}{l}\text { Drypetes roxburghii } \\
\text { (Euphorbiaceae) }\end{array}$ & Megamalai & Elev: 4995ft N: 09?39.835' E:077?21.763' \\
\hline 10 & $\begin{array}{l}\text { Alseodaphne somecarpifolia } \\
\text { (Lauraceae) }\end{array}$ & Pudhukadu & Elev: 5207ft N: 09?38.264' E:077?21.847' \\
\hline 11 & Ficus racemosa (Moraceae) & Kardana cave & Elev: $3709 \mathrm{ft}$ N: 09?41.610' E:077?24.232' \\
\hline 12 & Ficus asperima (Moraceae) & Highwavys & Elev: 4852ft N:09?39.872' E:077?21.747' \\
\hline 13 & Ficus beddomei (Moraceae) & Maharaja mettu & Elev: 5096ft N:09?38.373' E:077?22.018' \\
\hline 14 & Ficus retusa (Moraceae) & Maharaja mettu & Elev: 5026ft N:09?38.130' E:077?21.488' \\
\hline 15 & $\begin{array}{l}\text { Syzygium tamilnadensis } \\
\text { (Myrtaceae) }\end{array}$ & Pudhukadu & Elev: 5009ft N: 09?38.380' E:077?22.011' \\
\hline 16 & Syzygium cumini $\quad$ (Myrtaceae) & Maharaja mettu & Elev: 5013ft N:09?38.300' E:077?21.392' \\
\hline 17 & $\begin{array}{l}\text { Erythrina indica } \\
\text { (Papilionaceae) }\end{array}$ & Megamalai & Elev: 4945ft N:09?38.780' E:077?21.642' \\
\hline 18 & Coffea arabica (Rubiaceae) & Kardana estate & Elev: $3608 \mathrm{ft} \mathrm{N}: 09 ? 41.626$ ' E:077?24.104' \\
\hline 19 & $\begin{array}{l}\text { Todalia asiatica(L.)Lam } \\
\text { (Rutaceae) }\end{array}$ & Pudhukadu & Elev: 5009ft N: 09?38.380' E:077?22.011' \\
\hline 20 & $\begin{array}{l}\text { Achronychia pedunculata } \\
\text { (Rutaceae) }\end{array}$ & Megamalai & Elev: 4746ft N:09?38.259' E:077?21.264' \\
\hline 21 & $\begin{array}{l}\text { Palaquium ellipticum } \\
\text { (Sapotaceae) }\end{array}$ & Kardana estate & Elev: 3877ft N:09?41.643' E:077?24.165' \\
\hline
\end{tabular}


fruits were held against the body by hind leg and also by forming a wing pouch to hold. After processing the seeds of the larger fruits were dropped undamaged under the feeding roost. The skin, some smaller seeds and fibrous components of the fruit were spitted. This cleaning up of the seeds not only provides mechanical inducement for the seed germination but also prevent fungal attack because of the complete removal of the fleshy pericarp and the salivary chemistry with weak acids and enzymes. While consuming, small seeded fruits were swallowed and were defecated during commuting flights or while roosting. The gut processes the seeds for quick germination (Douglas 1979; Thomas 1991; Taylor et al. 1997; Lobova et al. 2003; Tang et al. 2005).

The faeces consisted of fruit pulp and seeds, with no visible change as a result of digestion, is an indication of quick defecation. Gut passage time in captive bats range from 15 to 20 minutes. The faeces are not cohesive so the seeds spread out in the air and fall separately. This feeding and foraging behavior underscores the superiority of $L$. salimalii over birds in seed dispersal and saves the seeds from interspecies competition. The seeds and the germinated seedlings of rare and endemic forest trees from the no cost nurseries (feeding roosts) of fruit bats can be used for reforestation programmes.

\subsection{Bat-Plant Interaction}

4.3.1 Bat Morphological Adaptation and Flight Performance Impact

Aerodynamic parameters of $L$. salimalii, helps to predict the different foraging modes associated with different resource partitioning (Strait and Overdorff 1996; Yamashita 1996). The sustained flight leads to quick faraway dispersal and pollination of plant species (Heithaus et al. 1975; Donald, 1991; Webb and Tidemann, 1996). L. salimalii has shorter, narrow wings with small wing area. Most frugivorous pteropodid bats are strong, direct fliers, not highly manoeuvrable, and fly with slow wingbeats (Kingdon
1974; Thomas 1975). For its size L. salimalii is responding to the pressure for fast commuting flight with the ability to carry fruit, but also for relatively rapid flight within clutter. Many authors assess manoeuvrability as a bat's ability to exploit its environment (Norberg and Norberg 1971; Andersson and Norberg 1981; Stockwell 2001; Swartz, Freeman and Stockwell 2003).

High wing loading is an indication of high flight speed (Patterson and Hardin 1969; Norberg and Rayner 1987), which helps L. salimalii to respond to the pressure of high-speed commuting flight. Minimum power speed (Vmp) $5.2 \mathrm{~m} / \mathrm{s}$ and maximum power speed range $(\mathrm{Vmr}) 6.9 \mathrm{~m} / \mathrm{s}$ promote sufficient foraging time. Bats foraging within clutter usually have to fly much slower with the minimum power speed, L. salimalii, rather with high flight speed manages with increased flight power, particularly during hovering for selection of fruit from the cluster.

The wing tip length ratio is much lower in $L$. salimalii and the tip area ratio is also lower. This means that the wingtip shape index becomes very high, higher than found in any other Pteropodids, and that the tips thus are very rounded. The roundness of the wing is mostly important for high manoeuvrability. It also increases agility at slower speeds enable the bat to fly with in clutter (Thollesson and Norberg 1991; Holderied and Jones 2009).

The combination of a low aspect ratio, a relatively short wingspan and a high wing loading is characteristic of $L$. salimalii to fly fast within vegetation with an amazing agility and even hover a few seconds in search of food similar to Rousettus, slightly larger species. The thrust generated by the flapping wings in $L$. salimalii permit the bat to turn without net loss of height, thus can make tight turns in the small space available (Jeyapraba 2008).

The ability to carry heavy prey or fruits is coupled with a low wing loading. Carnivorous species among microchiropteran bats carry prey which weighs half their own weight (Jones 1995). L. Salimalii, with 21.5 
$\pm 1.5\left(\mathrm{NM}^{-2}\right)$ wingloading has been observed to carry fruits up to $20 \%$ of its body mass. Fruits up to $28 \mathrm{gm}$ were carried away to the feeding roost for processing and consumption at leisure. L. salimalii being the only flying mammlian species found at higher elevations of the rain forest involved in carrying large fruits and seeds of Elaeocarpus sp. Palaquium ellipticum, Cullenia exillarata; with hard nuts.

\subsection{Chiropterophilic Plant Architecture and Phenology}

The tree architecture, fruit morphology and reproductive phenology are mainly responsible for species specific selection of feeding plants and their dispersal. Only a few plants maintain a year-round crop fruits with sufficient quantity and quality to sustain this bat population. The plant species in general offer two types of fruiting strategies. "Big bang" trees produce thousands of fruits over a limited time. But among them, individual trees fruits asynchronously, for example Ficus sp. "Steady state" trees provide few ripe fruits over a long period of time. In general the L. salimalii rely heavily on Ficus $s p$ in their diet. According to Wendeln et al., (2000) diet of mixed Ficus $s p$. can provide bat with the entire nutrient they require. The fortnight food item sample obtained were dominated with Ficus sp. and fig spit out pellets were collected through out the year. Where as other fruits are seasonal. L. salimalii commonly fly around the crowns of tall fruiting trees. By repeated circling the bat selects the ripened fruit from the clusters and seize them while on flying. The matured ripenned fruits easily wither/come off. Most of the core species of $L$. salimalii borne on branches or branchlets with defoliation adapted to be deciduous during fruiting. This enables the bats to collect the without much hindernce. If the fruit clusters are in the interior of the crown, the bats sometime cling to the branches while seizing the fruits.

\subsection{Bat Pollination and Its Impact on Propagation}

Bat pollination is a phenomenon restricted to the tropics and sub tropics. Observations indicate that in addition to fruits, the bats feed pollen and nectar for their basic mineral requirement and energy resource (Barclay, 1994 and 1995). Flowers pollinators by bats exhibit a number of characteristic adaptations collectively described as "syndrome of chiropterophily" (Vogel 1968, 1969a, b; Faegri and van der pij1 1971; Dobat 1985). Major characteristics of bat-pollinated flowers exhibit nocturnal anthesis and the flowering chronology correlates with bat activity, often limited to single night. Shape and sturdiness (brush or bell like flowers relate to the size of a head mask for a bat); production of larger quantity of nectar and pollen; an intense typical scent with strong odour and unpleasantly stale (Vogel 1969a; Bauer 1991; Bestmann et al., 1997; Helversen et al., 2000); inconspicuous colors (white or green or brown or brownish red) (Jacobs, 1992 and 1993) and a freely exposed position on the plant specifically to attract the fruit bats. Even though Eonycteris spelaea the only nectar feeder of southern Western Ghats benifits pollination, the documented results show Latidens also visit Chiropterophilic flowers to get balanced diet. Latidens with its long snout and bristly toung were able to lap up nectar and pollen and pollinate flowers. Thus involve in cross-pollination via nectar feeding (Moseley, 1879; Banack, 1998; Fleming and Estrada, 1996; Shilton et al., 1999; Godinez - Alvarez et al., 2002). Mostly the bat pollinated flowers are almost exclusive to bats because no other visitors are attracted or incapable of appreciable pollen transfer purely on mechanical grounds. For the propagation of such plant species the bat pollination is indispensable.

\subsection{Bat-Plant Chemical Interaction}

Earlier studies of Vanitharani on bat-plant chemical signaling revealed (Vanitharani 2011, Vanitharani and Pandian 2012), major composition of bat's saliva and faeces are a variety of alkanes. These alkanes perform significant role in seed germination with the combination of aliphatic compounds and alcohols 
(Sengupta, 2005). Methane, Decane and Cosane derivatives of the bat samples improves the texture of the seed for quick germination, increases adsorption by increasing the invasion capacity of nanopores in seeds (Stoner, 2004, Galarneau, 2008). Alcohols in general supports cellular regeneration (Trubin, 2006). The weak acids composition salival and gut promote seed desiccation tolerance. Aliphatic acid compounds present in the gut act as a coenzyme which helps the seed to recover dormancy and improves catabolic activity during germination. The aromatic compounds prevent the seeds form insect predation (Graven, 1996). Bat treated seeds with salivary phosphatase enzyme enables the seed to access organically bound phosphate nutrients.

Smoke treatment, hot/cold water soaking, acid treatment promote seed germination a bit early than the estimated time. Latidens processed seeds get similar high carbon compound treatment by passing through the gut, seed retention in the gut of bat and the buccal cavity give similar heat inducement treatment to the seeds. Studies of Bizerril and Raw 1998; Iudica and Bonaccorso 1997 also confirmed the fruit bat's gut and the salivary juices processes the seed and facilitates quick germination.

\section{Conclusions}

L. salimalii is a reliable propagator of few endemic trees of southern Western Ghats and plays a significant role in the evolution and maintenance of the tropical forest ecosystem. The present report provides research documentation about the splendid role of $L$. salimalii in the evergreen forest ecosystem. The study has also revealed $L$. salimalii is morphologically adapted to play a major role in seed dispesal and pollination. Seed dispersal is a prerequisite for the continuation of these tree species. The bat's species specific dietary preferences immensely help to restore and bring back the natural forest community structures. The existing undisturbed tropical forest and the sustainable fruiting trees of southern Western Ghats sincluded in two of the W.W.F. Global 200 Ecoregions, selected for their outstanding biodiversity (no. 20: south Western Ghats moist forests and no. 171: Western Ghats Rivers and Streams) $\}$ is the prerequisite for the existence of endemic endangered L. salimalii in southern Western Ghats. The study can give high recommendation to the Indian government to revise the Indian Wildlife Protection Act 1972 (categorized fruit bat species as VERMIN under Schedule V) and give conservation support to these valuable partner in the management of forest ecosystem.

\section{Acknowledgements}

Special thanks to Tamil Nadu Forest Department for granting permission to conduct this study. I am grateful to then Conservator of Forests, District Forest Officers and Range officers for given me filed support and logistical advice during the study. I specially acknowledge my research students and local tribes for their help with fieldwork in the forest interiors during night hours. I would like to extend my grateful thanks to the Whitley Foundation for the award of Rufford Small Grants, which serves to support continuing bat research and conservation initiatives in the southern Western Ghats. I am also grateful to Dr. V. Chelladurai (Retd.) of the Survey of Medicinal Plants Unit, Siddha, Tamil Nadu for his assistance in identifying seeds and seedlings from the feeding roosts. 